2. Consider the integral

$$
\begin{aligned}
I & \equiv \int_{0}^{n} x(x-1)(x-2) \ldots(x-n) d x \\
& =\left\{\int_{0}^{\frac{n}{2}}+\int_{\frac{n}{2}}^{n}\right\} x(x-1)(x-2) \ldots(x-n) d x ;(\text { Put } y=n-x) \\
& =\int_{0}^{n} x(x-1) \ldots(x-n) d x-(-)^{n} \int_{0}^{\frac{n}{2}} y(y-1) \ldots(y-n) d y \\
& =0, \text { if } n \text { is even. } \ldots \ldots \ldots(1)
\end{aligned}
$$

Now let $x(x-1) \ldots(x-n)=a_{1} x+a_{2} x^{2}+\ldots+a_{n+1} x^{n+1}$. Then if $n$ is even, we have a consistent set of $(n+1)$ equations in the $a$ 's,

$$
\begin{aligned}
& 0=a_{1}+a_{2}+\ldots \ldots+a_{n+1} \\
& 0=2 a_{1}+2^{2} a_{2}+\ldots \ldots+2^{n+1} a_{n+1}, \\
& \ldots \ldots \ldots \ldots \ldots \ldots \ldots \\
& 0=n a_{1}+n^{2} a_{2}+\ldots \ldots+n^{n+1} a_{n+1},
\end{aligned}
$$

and by (1), $0=\frac{n}{2} a_{1}+\frac{n^{2}}{3} a_{2}+\ldots+\frac{n^{n+1}}{n+2} a_{n+1}$,

and hence the determinant of the system is zero, which is Jung's result.
A. C. Aitken.

\title{
On the Roots of a Symmetrical Determinant.
}

The six values of $x$ which make the determinant

$$
\Delta=\left|\begin{array}{cccccc}
x & 1 & . & . & . & . \\
1 & x & 1 & . & . & . \\
. & 1 & x & 1 & . & . \\
. & . & 1 & x & 1 & . \\
. & . & . & 1 & x & 1 \\
. & . & . & . & 1 & x
\end{array}\right|
$$

vanish, are $-2 \cos \frac{\pi}{7},-2 \cos \frac{2 \pi}{7}, \ldots,-2 \cos \frac{6 \pi}{7} . \quad$ In general, the $n$ values of $x$ which make the corresponding determinant of order $n$ vanish are given by $-2 \cos \frac{r \pi}{n+1}, r=1,2, \ldots, n$. Each determinant has a diagonal filled with $x$ 's, bordered by adjacent parallels where each element is unity; and all other elements are zero. 
$\mathrm{xvi}$

The above result is no novelty, but the following geometrical proof may be of interest. Let $n+1$ be a prime number, such as 7 , and let $a, b, c, d, e, f$ denote the distances of a vertex $O$, of a regular heptagon $O A B C D E F$, from the other six vertices, taken in cyclic order from $O$. Let $\alpha$ be the angle subtended at $O$ by each of the equal sides $O A, A B$, etc., so that $a$ is $\frac{\pi}{7}$, and in general is $\frac{\pi}{n+1}$.

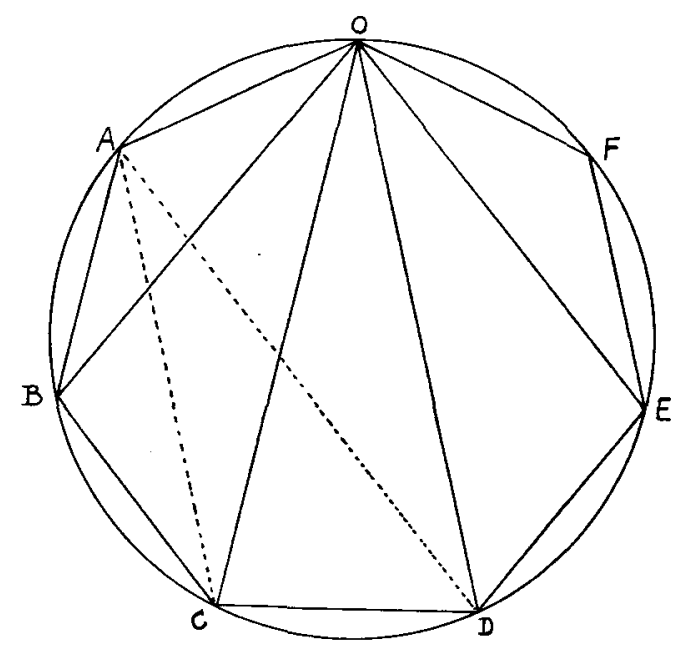

Since $O A=C D$, it follows that $A C$ is parallel with $O D$, and $A D=O C$. Hence, by projection,

or

$$
\begin{aligned}
& (O C+A D) \cos a=O D+A C=O D+O B, \\
& O B-2 O C \cos a+O D=0 .
\end{aligned}
$$

By repeating this process we obtain the six equations

$$
\begin{aligned}
-2 a \cos \alpha+b & =0, \\
a-2 b \cos \alpha+c & =0, \\
b-2 c \cos a+d & =0, \\
c-2 d \cos a+e & =0, \\
d-2 e \cos \alpha+f & =0, \\
e-2 f \cos \alpha & =0 ;
\end{aligned}
$$

whence, by elimination, $\Delta=0$ when $x=-2 \cos \alpha=-2 \cos \frac{\pi}{7}$.

The same argument proves that $-2 \cos \frac{2 \pi}{7}$ also is a root of the 
equation $\triangle=0$, if $O B D F A C E$ is taken as the heptagon, and the angle $B O D$ is taken as $a$. Similarly the heptagon $O C F B E A D$ leads to $-2 \cos \frac{3 \pi}{7}$, and so on, until six different cases have been identified.

Manifestly the process will apply, giving $n$ roots of $\Delta=0$, whenever $n+1$ is prime. If $n+1$ is composite the theorem is still true, as the argument remains applicable; but the proof is not so pretty, as several of the polygons degenerate.

The theorem may be stated otherwise:

The latent roots of the matrix of order $n$

$$
\left[\begin{array}{cccccc}
. & 1 & . & \cdot & . & \ldots \\
1 & \cdot & 1 & . & . & \ldots \\
\cdot & 1 & . & 1 & . & \ldots \\
\cdot & \cdot & 1 & . & 1 & \ldots \\
\ldots & \ldots & \ldots & \ldots & \ldots &
\end{array}\right]
$$

are given by $\quad-2 \cos \frac{r \pi}{n+1}, \quad r=1,2, \ldots, n$.

If the matrix is of infinite order its latent roots are all real, lying densely between -2 and +2 .

H. W. TURNBULL. 